\title{
Connections between Hadronic Masses in the One Hand and between Fundamental Particle Masses in the Other Hand
}

\author{
Boris Tatischeff \\ IPNO, CNRS/IN2P3, Université Paris-Sud, Universté Paris-Saclay, 91406 Orsay Cedex, France \\ Email: tati@ipno.in2p3.fr
}

\begin{abstract}
The oscillation symmetry is used to study the connections between masses and widths of a selection of the following states studied separately: mesons, baryons, nuclei, and hypernuclei. It is also applied to study the connection between leptonic, quark and boson masses and widths. With the exception of $\mathrm{M} \approx 0$ mass particles, all the fundamental particle masses are fitted by a single distribution inside the oscillation symmetry.
\end{abstract}

Keywords: oscillation symmetry, particles, hadrons, nuclei, masses, widths

\section{Introduction}

The oscillation symmetry, was extended recently, from the classical world to the quantum one. Indeed here the masses are solution of the Schrödinger eq., containing kinetic and potential interactions, as are the oscillations of pendulums or springs. This was observed in particle and nuclei level masses [1]. Then such oscillation property was also observed in different other data, suggesting that this property is rather general in nature. The oscillation property is observed, provided that the studied objects are submitted to opposite interactions and whatever the nature of the interactions involved. Opposite interactions act always when bodies result from smaller bodies combinations. Otherwise these bodies will either disintegrate or compress themselves up to loose their initial state as do plasmas for example. Such situation exists often in nature: for example nuclei made with nucleons, or nucleons with quarks.

In different situations, opposite interactions act on bodies which are not the object of smaller body combinations. So it was shown that such symmetry applies in many astrophysical properties, since the corresponding bodies are submitted to opposite gravitational forces and centrifugal forces related to their kinetic energies [2] [3].

The present paper is limitated to some nuclei and hadronic and fundamental particle masses. We start to classify the studied masses in increasing order. The possible oscillations in the mass spectra are studied using the following relation:

$$
m_{(n+1)}-m_{n}=f\left[\left(m_{(n+1)}+m_{n}\right) / 2\right]
$$

where $m_{(n+1)}$ corresponds to the $(\mathrm{n}+1)$ mass value. Two successive mass differences are therefore plotted versus their corresponding mean values. Such values will be named "mass data" in the continuation of this work.

A normalised cosine function is used for the fits of the data:

$$
\Delta M=\alpha\left(1+\cos \left(\left(M-M_{0}\right) / M_{1}\right)\right) * \exp \left(\beta .\left(M-M_{C}\right)\right)
$$

where $\mathrm{M} / \mathrm{M}_{1}$ is defined within $2 \pi$. The oscillation period is $\mathrm{P}=2 \pi M_{1}$.

Although there is no clear justification to observe oscillations on the widths of particles, and of nuclear levels, it was already shown that they are also observed. The oscillations will be therefore looked for in figs. showing widths versus masses. Thus in equation (2) $\Delta \mathrm{M}$ corresponds to the ordinate and $\mathrm{M}$ to the abscissa. When fitting the mass data, $\Delta \mathrm{M}$ corresponds to $\mathrm{m}(\mathrm{n}+1)-\mathrm{m}(\mathrm{n})$ and $\mathrm{M}$ corresponds to $(\mathrm{m}(\mathrm{n}+1)$ $+\mathrm{m}(\mathrm{n})) / 2$. For the other figures, $\Delta \mathrm{M}$ correponds to the width and $\mathrm{M}$ to the mass.

$\mathrm{M}$ is the variable, $\alpha, \beta, M_{C}$ and $M_{1}$ are the fit parameters. $\mathrm{M}_{0}$ is arbitrary and taken to 0 . The fits are obtained using relation (2) using most of the time three (in a few cases two) parameters reported 
in Table 1 . In case of large masses $\mathrm{M}$, a mass $\mathrm{M}_{C}$ is sometimes introduced in order to replace $\beta \mathrm{M}$ by $\beta(\mathrm{M}-\mathrm{MC})$ and avoid a very small $\beta$ value. These $\mathrm{M}_{C}$ are arbitrary and are not therefore considered as a fitted parameter. They are however given in Table 1 since they are bound to the corresponding $\beta$.

There is no color meaning in figs. showing data with a single color spot. Different color spots inside the same fig. show different data.

\section{Mesonic Masses}

Some data and fits have been shown in [1] and will not be reproduced here. They correspond to masses and widths of unflavoured mesons and strange mesons. The results corresponding to different data [4] are studied here.

Fig.1 shows the mass data for $\omega$ and $\rho$ mesons in inserts (a) and (b) [4]. The corresponding widths data are shown in inserts (c) and (d). New observed $\omega$ states $\mathrm{X}(2040)$ and $\mathrm{X}(2240)$ [5] are introduced in Fig 1. A good agreement is observed in all data, except for the width of the lowest $\omega$ mass.
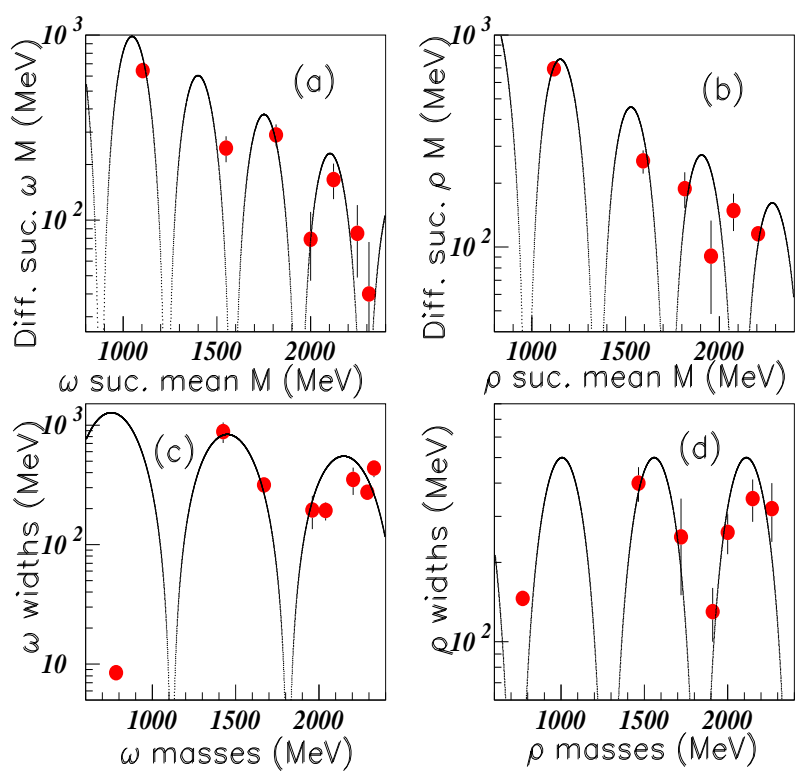

Figure 1. Color on line. Inserts (a) and (c), show respectively the mass data and the width data for $\omega$ mesons. Inserts (b) and (d), show respectively the mass data and the width data for $\rho$ mesons. (See text).

Fig. 2 shows in insert (a) the difference between successive $\mathrm{D}^{ \pm}$masses [4] [6] versus their corresponding mean values. Insert (b) shows the $\mathrm{D}^{ \pm}$widths versus their masses. Inserts (c) shows the mass data for $\mathrm{D}_{S}^{ \pm}$ [4] [7]. Insert (d) shows the mass data for $\mathrm{B}^{ \pm}$mesons in full purple circles and $\mathrm{B}_{S}$ mesons in full bluesky squares. The same fit describe the data for $\mathrm{B}^{ \pm}$and $\mathrm{B}_{S}$ meson data. A good agreement is observed between data and fits in all four inserts.

Fig. 3 shows the data and fits for $0^{-}\left(1^{--}\right)$charmonium and bottomonium mass and width data. In inserts (a) and (b) the differences between successive masses are plotted versus their corresponding mean values. In inserts (c) and (d) the full (electromagnetic $\Gamma_{e e}$ ) widths are plotted versus the corresponding masses. All these data are well fitted, including the very small widths for levels which masses are lower than their strong disintegration thresholds. These thresholds are $\mathrm{M}_{t h}=3740 \mathrm{MeV}$ for $\mathrm{c} \bar{c}$ mesons and $\mathrm{M}_{t h}=10560 \mathrm{MeV}$ for $\mathrm{b} \bar{b}$ mesons. We observe the same period for $\mathrm{c} \bar{c}$ and $\mathrm{b} \bar{b}$ width distributions (see Table 1) and close periods for their mass data. 

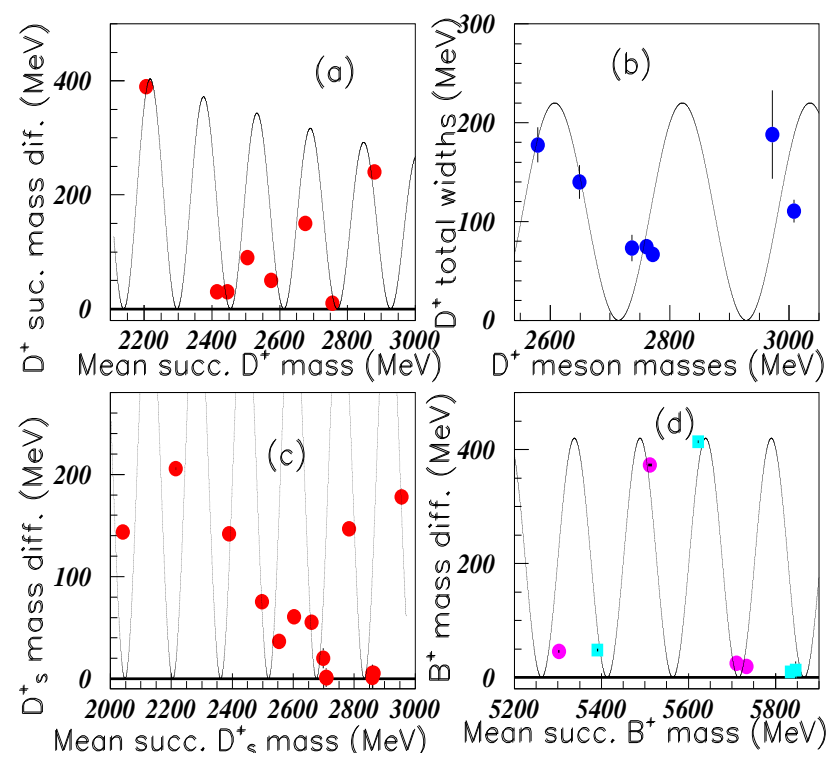

Figure 2. Color on line. Inserts (a), (b), and (c) show respectively the mass data for $\mathrm{D}^{+}$, the width data for $\mathrm{D}^{+}$, and the mass data for $\mathrm{D}_{S}^{+}$mesons. Insert $(\mathrm{d})$ shows the mass data for B and $\mathrm{B}_{S}$ mesons. (See text).
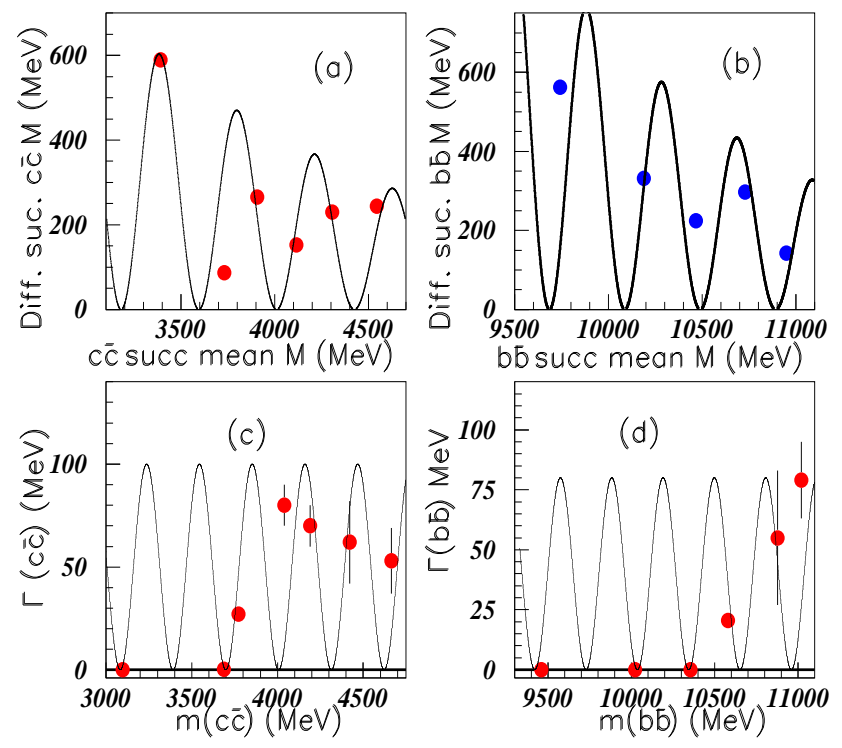

Figure 3. Color on line. Inserts (a) and (b) show respectively the mass data and fits for $c \bar{c}$ and $b \bar{b} \quad 0^{-}\left(1^{--}\right)$ mesons. Inserts (c) and (d) show respectively the width data and fits for $c \bar{c}$ and $\mathrm{b} \bar{b}$ mesons. (See text).

\section{Baryonic Masses}

The data and fits corresponding to $\Delta$ baryons were shown in [1]. Here data corresponding to different baryons are studied.

Fig. 4 shows the data and fits for $\Sigma$ baryons [4]. The results for mass data with spins $1 / 2$ and $3 / 2$, are shown in inserts (a) and (b), respectively. The results for widths with spins $1 / 2$ and $3 / 2$, are shown in inserts (c) and (d). The periods for the two spin data are (nearly) the same, but larger for the width than for the mass, unlike they are for the meson data shown in fig. 3. 


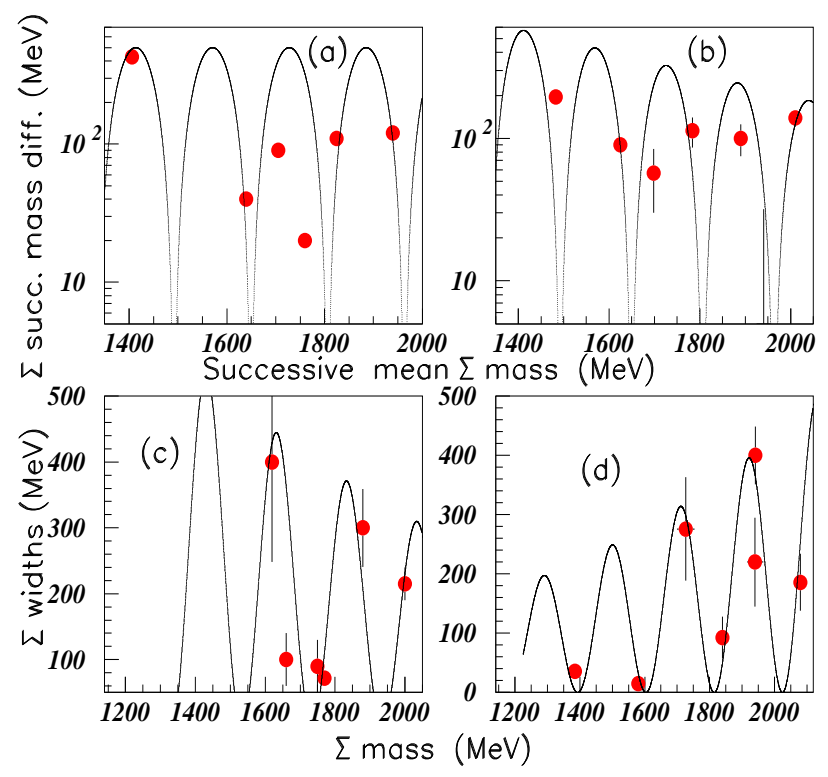

Figure 4. Color on line. Data and fits for $\Sigma$ baryons. The mass studies are shown in inserts (a) $\mathrm{J}=1 / 2$ and (b) $\mathrm{J}=3 / 2$. The widths are shown in inserts (c) $\mathrm{J}=1 / 2$ and (d) $\mathrm{J}=3 / 2$. (See text).
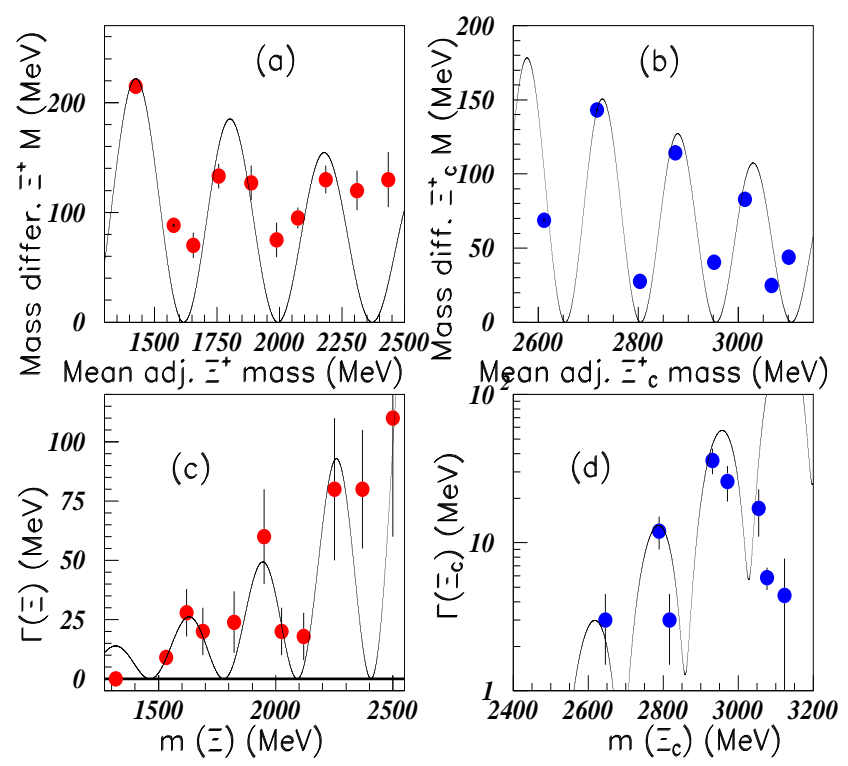

Figure 5. Color on line. Inserts (a) and (b) show respectively the mass data for the $\Xi$ and $\Xi_{C}$ baryons. Inserts (c) and (d) show respectively the width data for the $\Xi$ and $\Xi_{C}$ baryons. (See text).

Fig. 5 shows the data and fits for $\Xi$ baryons in inserts (a) and (c) (red full circles data) and $\Xi_{C}$ baryons in inserts (b) and (d) (blue full squares data). Inserts (a) and (b) study masses, when inserts (c) and (d) study widths.

Fig.6 shows the data and fits for $\Lambda_{C}$ (full red circles) and $\Sigma_{C}$ (full blue squares) baryons. Masses (widths) are shown in insert (a) ((b)). These $\Lambda_{C}$ and $\Sigma_{C}$ data are well fitted by the same distributions. 


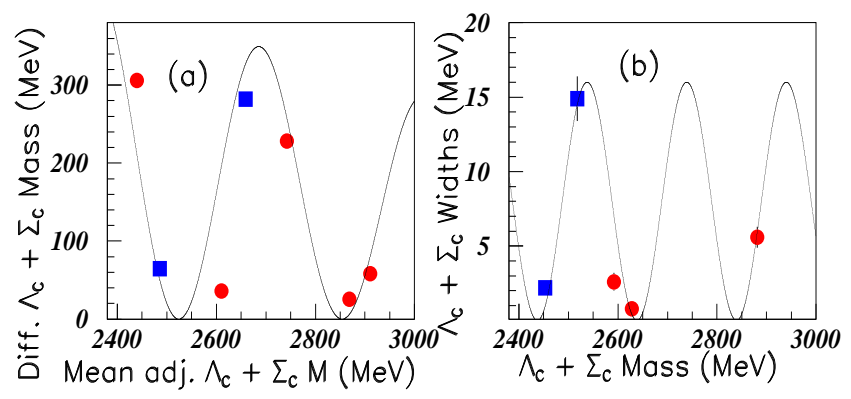

Figure 6. Color on line. Inserts (a) and (b) show respectively the mass data for the $\Lambda_{C}$ and $\Sigma_{C}$ baryons. Inserts (c) and (d) show respectively the width data for the $\Xi$ and $\Xi_{C}$ baryons. (See text).

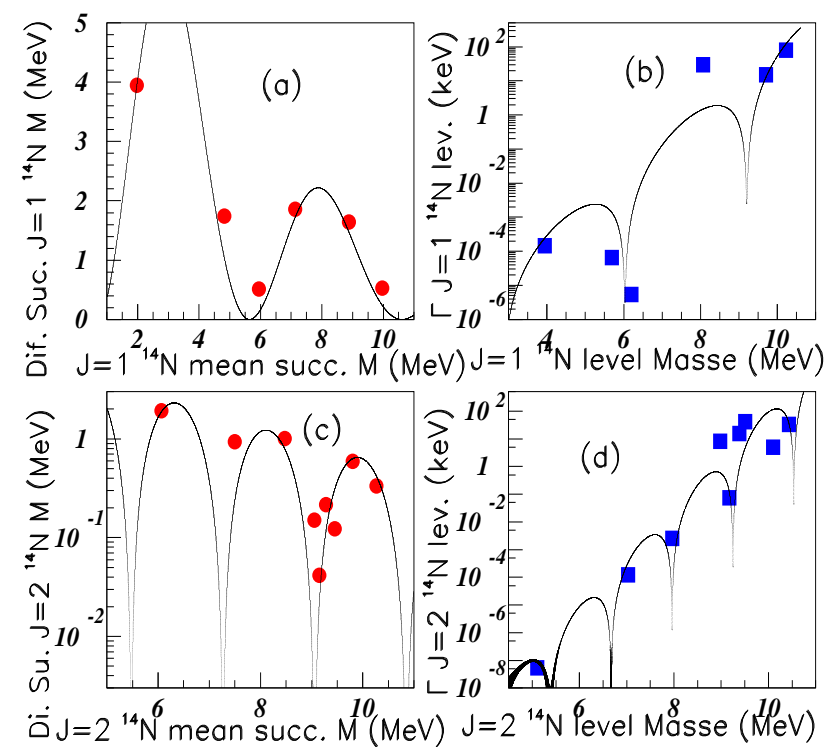

Figure 7. Color on line. Insert (a) ((c)) shows the mass data for the excited levels of ${ }^{14} \mathrm{~N}$ with the spin $\mathrm{J}=1$ $(\mathrm{J}=2)$. Insert (b) ((d)) shows the width data for ${ }^{14} \mathrm{~N}$ levels and $\mathrm{J}=1(2)$. (See text).

\section{Nuclei Masses}

The nuclei level widths are reported with different units: $\tau_{m}, \mathrm{~T}_{1 / 2+}$, or $\Gamma$. They are all converted in kev (mev). They are sometimes unknown. The values of the parameters describing the fits are reported in Table A2.

Fig. 7 shows the data and fits for ${ }^{14} \mathrm{~N}$ [8]. The mass data for $\mathrm{J}=1(\mathrm{~J}=2)$ levels are plotted in insert (a) ((c)) with full red circles. The widths data for $\mathrm{J}=1(\mathrm{~J}=2)$ levels are plotted in insert (b) ((d)) with full blue squares. The ratios of mass periods versus width periods are rather close for both spin data, namely close to 2.6. The mass data decrease for larger level masses, when the width data increase.

Fig. 8 shows the total widths of ${ }^{15} \mathrm{O}$ excited state levels versus the corresponding masses [8]. Inserts (a), (b), and (c) show respectively the data for the spins $\mathrm{J}=1 / 2,3 / 2$, and $5 / 2$. The threshold to proton decays is close to $7.5 \mathrm{MeV}$, the decay widths for lower excited levels are very low and therefore omitted from this fig. Indeed the fundamental state $\mathrm{J}=1 / 2$ is quasi stable, the width of the next $\mathrm{J}=1 / 2$ level at $5.183 \mathrm{MeV}$ is very small and equals $9.1 \mathrm{meV}$. The oscillating periods decrease with increasing spin data.

A nice agreement is observed between data and fits.

Fig. 9 shows the mass data [9] for the excited states of $\mathrm{J}=2$ levels of ${ }^{26} \mathrm{Mg}, \mathrm{J}=5 / 2$ levels of ${ }^{27} \mathrm{Al}, \mathrm{J}=2$ levels of ${ }^{28} \mathrm{Si}$, and $\mathrm{J}=7 / 2$ levels of ${ }^{29} \mathrm{Si}$ nuclei respectively in inserts (a), (b), (c), and (d). 


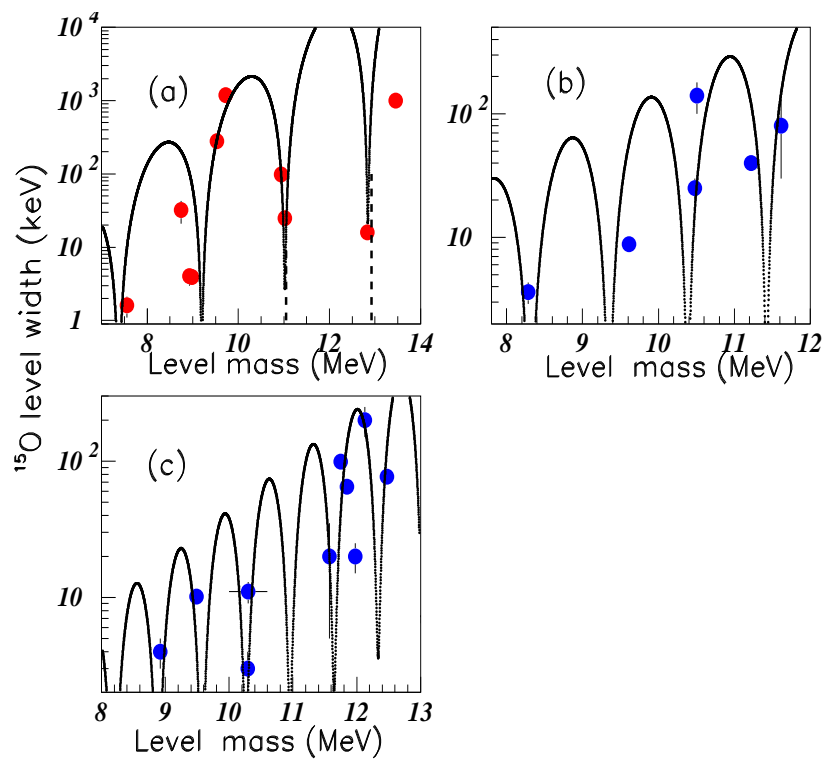

Figure 8. Color on line. Total widths of ${ }^{15} \mathrm{O}$ excited state levels versus the corresponding masses. Inserts (a), (b), and (c) show respectively the data for the spins $\mathrm{J}=1 / 2,3 / 2$, and $5 / 2$. (See text).

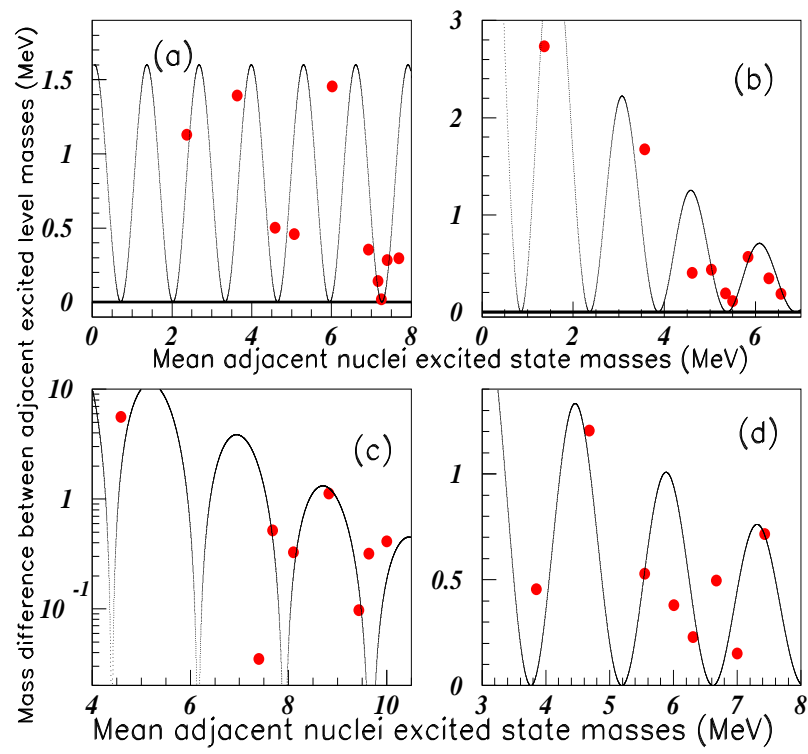

Figure 9. Color on line. Mass data of nuclei excited state levels. Inserts (a), (b), (c), and (d) show respectively the data for $\mathrm{J}=2$ levels of ${ }^{26} \mathrm{Mg}, \mathrm{J}=5 / 2$ levels of ${ }^{27} \mathrm{Al}, \mathrm{J}=2$ levels of ${ }^{28} \mathrm{Si}$, and $\mathrm{J}=7 / 2$ levels of ${ }^{29} \mathrm{Si}$ nuclei. (See text).

Fig. 10 shows the total widths of ${ }^{208} \mathrm{~Pb}$ excited state levels versus the corresponding masses [10]. Inserts (a), (b), (c), and (d) show respectively the data for the spins $\mathrm{J}=3,4,5$, and 6 . The width periods for odd nuclei are somewhat larger than the width periods for even nuclei. A nice agreement is observed between data and fits, mainly in inserts (a), (c), and (d).

Fig. 11(a) shows the variation of the hadronic family periods. Full marks show the periods obtained in the present paper, empty marks correspond to results of previous studies. Red circles show the meson results, blue squares show the baryon results. They are both fitted by relation (2) and the same two parameters, namely $\mathrm{P}=458.7 \mathrm{MeV}$, and $\alpha=220 \mathrm{MeV}$. 

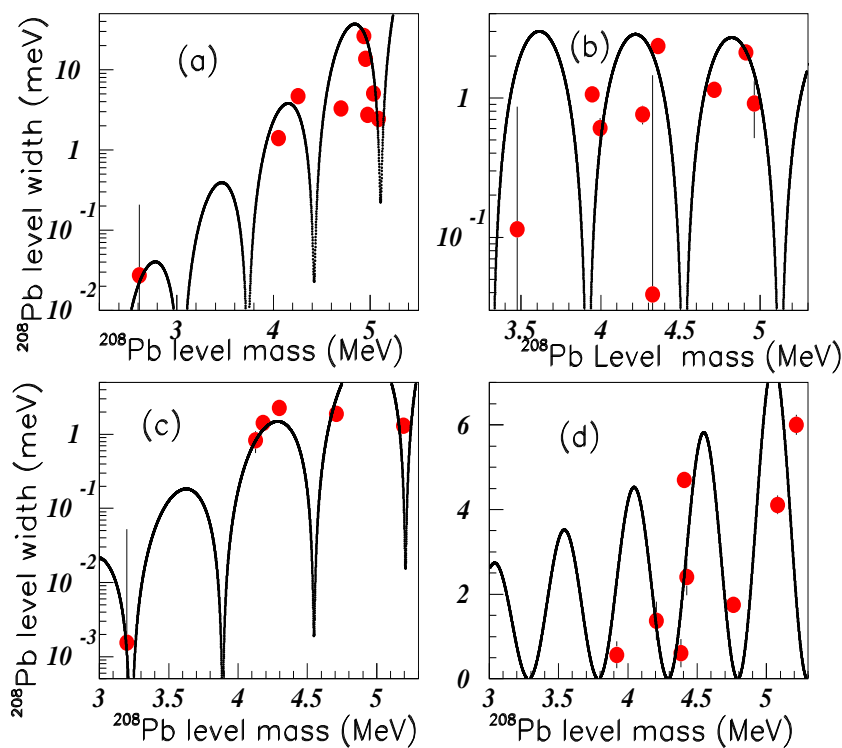

Figure 10. Color on line. Total widths of ${ }^{208} \mathrm{~Pb}$ excited state levels versus the corresponding masses. Inserts (a), (b), (c), and (d) show respectively the data having spins $\mathrm{J}=3,4,5$, and 6 . (See text).

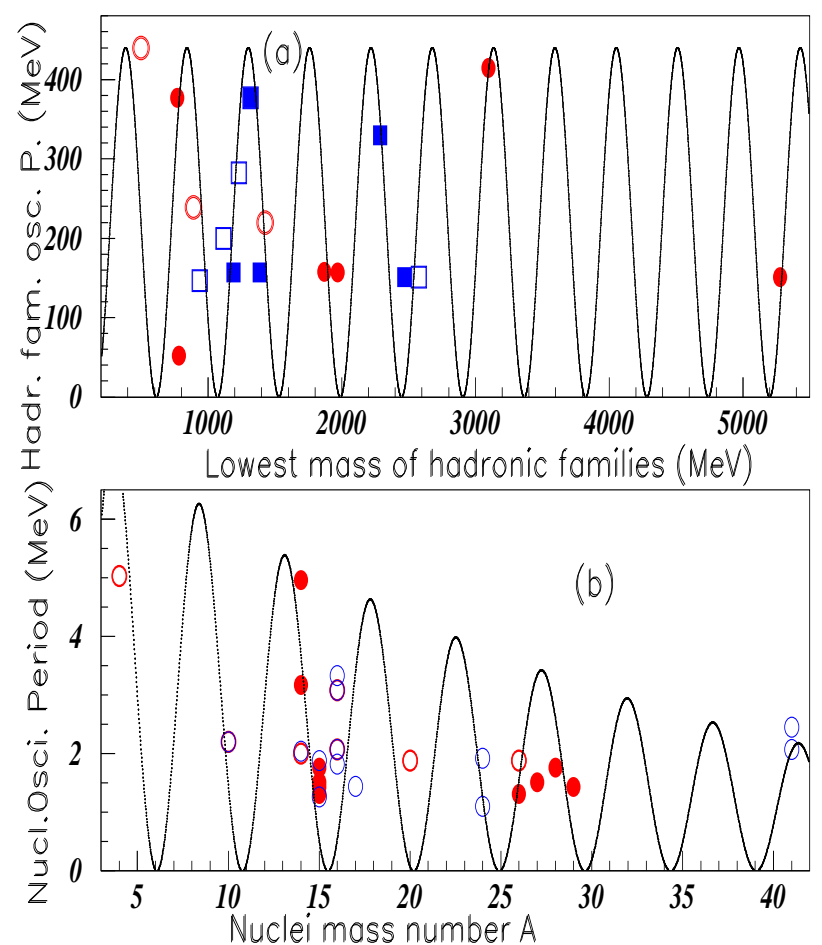

Figure 11. Color on line. Insert (a) shows the hadronic oscillation periods versus the fundamental corresponding masses. Insert (b) shows the nuclei oscillation periods versus the corresponding atomic mass number A. (See text).

Fig. 11(b) shows the nuclei periods of oscillation versus their atomic number A. Here again full (empty) circles show the present (previous) results. The dispersion of previous period values for the same 
A, corresponds to different spin data studies [1]. The fit is obtained with $\mathrm{P}=4.71 \mathrm{~A}, \alpha=4.1 \mathrm{MeV}$ and $\beta=-0.032 \mathrm{~A}^{-1}$.

\section{$5 \quad$ Hypernuclei Masses}

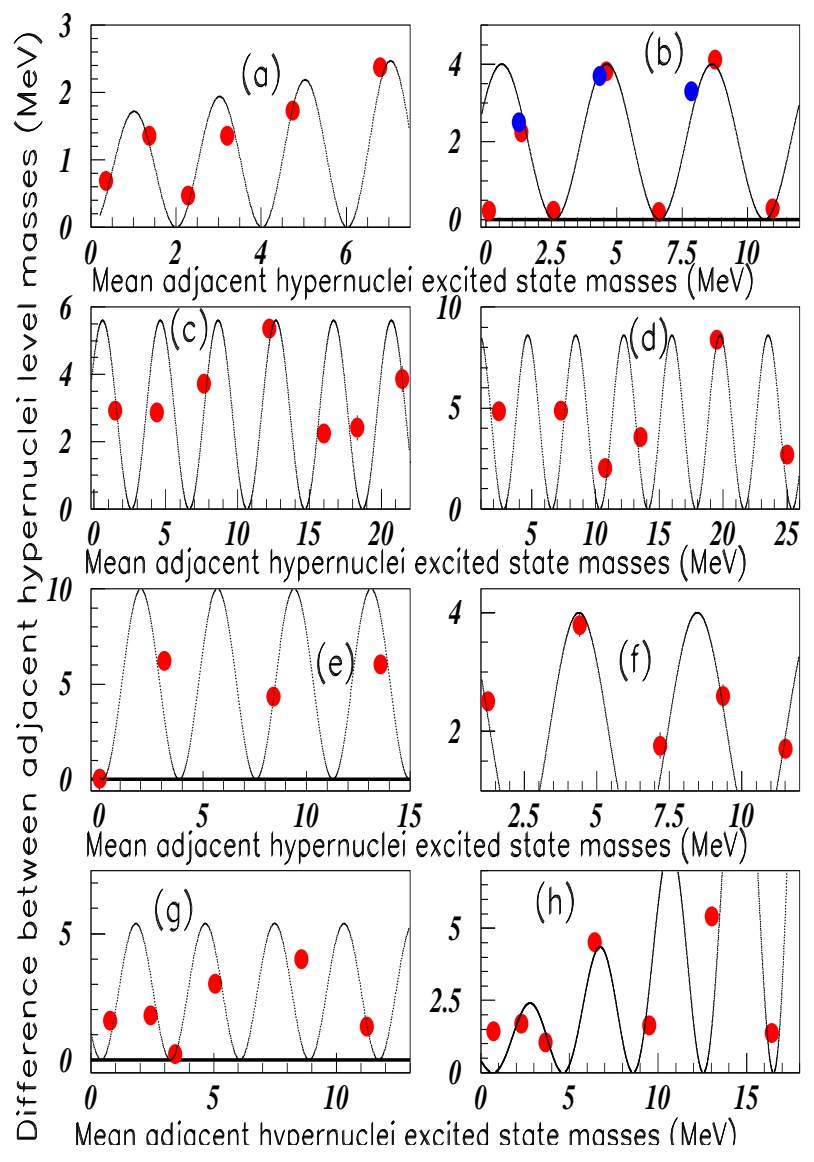

Figure 12. Color on line. Hypernuclei mass data versus their corresponding masses. Inserts (a), (b), (c), (d), (e), (f), (g), and (h) show data and fits for ${ }_{\Lambda}^{7} \mathrm{Li},{ }_{\Lambda}^{10} \mathrm{~B}$ (red data) and ${ }_{\Lambda}^{11} \mathrm{~B}$ (blue data), ${ }_{\Lambda}^{9} \mathrm{Be},{ }_{\Lambda}^{13} \mathrm{C},{ }_{\Lambda}^{16} \mathrm{O},{ }_{\Lambda}^{12} \mathrm{C},{ }_{\Lambda}^{51} \mathrm{~V}$, and ${ }_{\Lambda}^{89} \mathrm{Y}$. (See text).

Fig.12 shows data and fits of eight hypernuclei in inserts (a) ${ }_{\Lambda}^{7} \mathrm{Li}$ [11], (b) ${ }_{\Lambda}^{10} \mathrm{~B}$ [11] in red data and ${ }_{\Lambda}^{11} \mathrm{~B}[11]$ in blue data, (c) ${ }_{\Lambda}^{9} \mathrm{Be}[11],(\mathrm{d}){ }_{\Lambda}^{13} \mathrm{C}[11]$, (e) ${ }_{\Lambda}^{16} \mathrm{O}[11],(\mathrm{f}){ }_{\Lambda}^{12} \mathrm{C}[12],(\mathrm{g}){ }_{\Lambda}^{51} \mathrm{~V}[12]$, and ${ }_{\Lambda}^{89} \mathrm{Y}[12]$. The data are very well fitted, except the first one in insert $(\mathrm{h})$.

The variation of the corresponding periods is shown in fig. 13

\section{Fundamental Particle Masses}

The masses are read in [4]. Four lepton $(\mathrm{e}, \mu, \tau, \nu)$ masses (drawn in the following figures in full blue squares) are used. All neutrinos masses are considered to be null. Six quark (u, d, s, c, b, and t) masses (in full red circles) are used from $\mathrm{m}_{u}=2.5 \mathrm{MeV}$ up to $\mathrm{m}_{t}=173.5 \mathrm{GeV}$. Several boson masses (in full green triangles) are used. They are three gauge boson masses ( $\gamma$, gluon, and graviton masses which are supposed to be null), $\mathrm{W}$ and $\mathrm{Z}$. The new boson at $\mathrm{m} \approx 17.5 \mathrm{MeV}$ which existence was recently strengthened [14] 


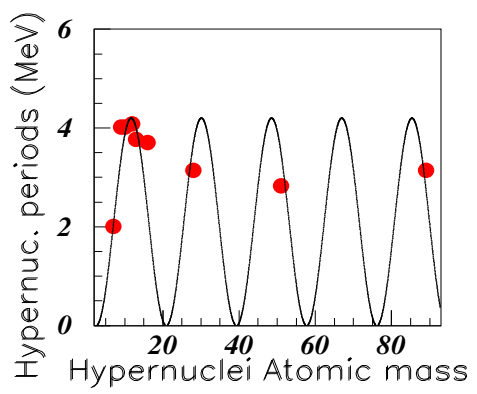

Figure 13. Color on line. Periods of hypernuclei mass oscillations versus their atomic mass A. (See text).

and theoretically predicted some years ago [15], is also considered, as well as the Higgs boson mass at $\mathrm{m}_{H}=125.1 \mathrm{GeV}$.

The data obtained using the three fundamental particle masses and relation (1) applied separately to each particle familly, are reported in fig. 14. The large mass variation between 0 up to $150 \mathrm{GeV}$ requires to divide the fig. in different inserts corresponding to different ranges and different mass scales.

Insert (a) of fig. 14 shows in linear scale the data up to $1000 \mathrm{MeV}$. Insert (b) shows the same data and fit as insert (a), except that the data are shown in log scale in both dimensions and up to $3200 \mathrm{MeV}$. It allows to better visualize the good agreement between low mass data and fit. The data in insert (c) show the large mass data. The drawn period in insert (c) is much larger (by a factor 103). The same period as in inserts (a) and (b) will give rise to the same agreement with data, as shown in insert (c), but with 103 more oscillations. We observe that all data are well fitted by a single distribution.

Fig. 15 shows the fundamental particle widths plotted versus the corresponding masses. Few widths of these particles, are known. The lepton widths as well as the quark widths are taken to be close to zero. The total width for $\mathrm{W}$ and $\mathrm{Z}$ bosons are known; the width for the 17 boson mass is very small $\Gamma=$ $3.9^{*} 10^{-5} \mathrm{eV}[16]$. The width for the "t" quark is known. The widths close to zero are drawn arbitrarily to $1.5 \mathrm{keV}$ in order to not increase the range of the figure, already large by more than nine orders of magnitude.

Fig. 15 is divided into two parts separated by a vertical line at $\mathrm{M}=60000 \mathrm{MeV}$. Table A2 shows successively the parameter values for the left then the right part of fig. 15. The period in the right part is increased by a factor 6 from the period of the left part. If keeping for the right part the period of the left part, the fit will be as good as the one shown, but we will obtain 6 times more oscillations.

So the data obtained using the relation (1), applied to fundamental particle masses, display an unexpected behaviour. In order to see if such behaviour is only the consequence of the particular relation (1), fig. 16 shows the variation of the mass ratios $m(n+1) / m(n)$ between successive masses versus again their corresponding mean value $[\mathrm{m}(\mathrm{n}+1)+\mathrm{m}(\mathrm{n})] / 2$. As in figs. 14 and 15 , red full circles correspond to quark data, blue full squares to lepton data and green full triangles to boson data. The same masses as used before are considered except the masses equal to zero. In this fig. the log-log scale involves to study the fit to data using a larger period after $\mathrm{M}=10000 \mathrm{MeV}$. The first period is equal to $\mathrm{P}_{1}=3141.59 \mathrm{MeV}$ $\left(1000^{*} \pi\right)$, the second period is larger by a factor $10\left(\mathrm{P}_{2}=31415.9 \mathrm{MeV}\right)$. Keeping the first period to large mass data will give rise to 10 times more oscillations and the same agreement with data. Seven data (over ten) are well described by a single fit. The agreement between data and fit is therefore not as good as with the relation (1).

\section{Conclusion}

The paper shows that mass data obtained using relation (1), as well as widths, exhibit regular oscillations. This property is applied for different families of mesons, baryons, nuclei and hypernuclei excited levels. These data are fitted with equation (2), most of the time using three parameters, and in a few cases two or four parameters. Although the data are generally well fitted by the equation (2), in a few cases a few number of data lie outside the fit. I do not know if this is due to an underestimation of the imprecision of the measurements, or some other cause. This oscillation symmetry can be used to tentatively predict 


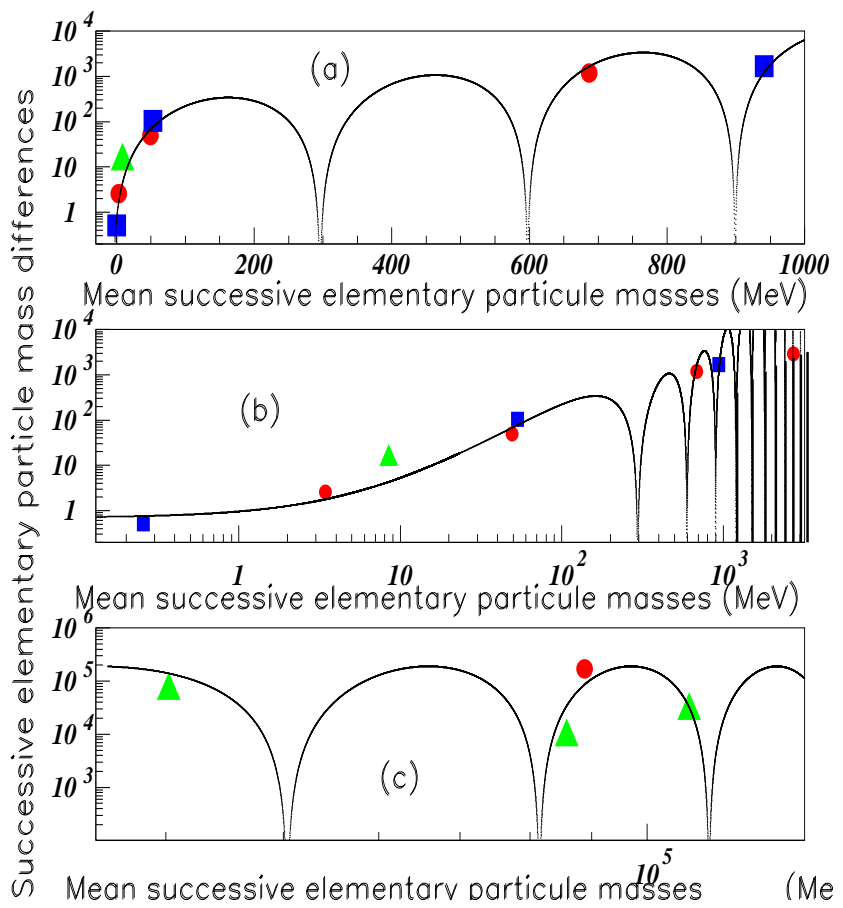

Figure 14. Color on line. Data obtained using the three fundamental particle masses, each of them being obtained using relation (1). (See text).

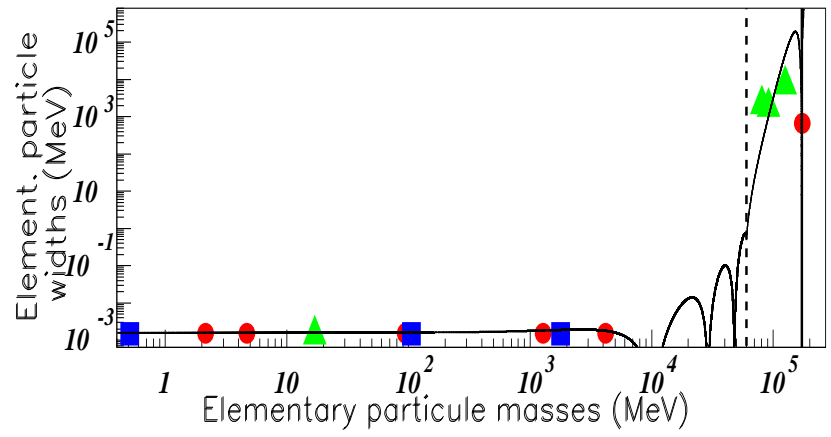

Figure 15. Color on line. Fundamental particle widths plotted versus the corresponding masses (See text).

new particle masses or other properties, if the unknown value is located somewhere inside the range of known data.

The oscillation symmetry is also well applied to fundamental particle masses. This work shows that they are connected through the symmetry of oscillation. This continuity is observed for data obtained with help of relation (1). So, using the simple oscillation symmetry formula, the same distribution describes the boson mass data, the lepton mass data and the quark mass data and therefore provides a connection between all them. Thus, contrary to the generally accepted view that the masses of fundamental particles have no relation between them, that property allows to observe that the fundamental particle masses are related through the oscillation symmetry.

This property allows us to question the possibility that these fundamental particles are themselves formed of smaller components. However "there is never been any evidence that they are made up of anything at all". 
Table 1. Parameters describing the fits of figs.1-5 showing the oscillation symmetry applied to several mesonic and baryonic masses. See text.

\begin{tabular}{ccccc}
\hline Fig. & $\alpha$ & $\beta\left(\mathrm{MeV}^{-1}\right)$ & $\mathrm{M}_{C}(\mathrm{MeV})$ & $\mathrm{P}(\mathrm{MeV})$ \\
\hline $1(\mathrm{a})$ & $2100 \mathrm{MeV}$ & -0.00138 & 0 & 351.9 \\
$1(\mathrm{~b})$ & $1900 \mathrm{MeV}$ & -0.00138 & 0 & 377 \\
$1(\mathrm{c})$ & $1000 \mathrm{MeV}$ & -0.0006 & 0 & 697.4 \\
$1(\mathrm{~d})$ & $250 \mathrm{MeV}$ & 0 & 0 & 552.9 \\
\hline $2(\mathrm{a})$ & $625 \mathrm{MeV}$ & -0.00051 & 0 & 157.7 \\
$2(\mathrm{~b})$ & $110 \mathrm{MeV}$ & 0 & 0 & 213.6 \\
$2(\mathrm{c})$ & $200 \mathrm{MeV}$ & 0 & 0 & 157.1 \\
$2(\mathrm{~d})$ & $210 \mathrm{MeV}$ & 0 & 0 & 150.8 \\
\hline $3(\mathrm{a})$ & $380 \mathrm{MeV}$ & -0.0006 & 3000 & 414.7 \\
$3(\mathrm{~b})$ & $615 \mathrm{MeV}$ & -0.0007 & 9200 & 402.1 \\
$3(\mathrm{c})$ & $50 \mathrm{MeV}$ & 0 & 0 & 307.9 \\
$3(\mathrm{~d})$ & $40 \mathrm{MeV}$ & 0 & 0 & 307.9 \\
\hline $4(\mathrm{a})$ & $250 \mathrm{MeV}$ & 0 & 0 & 157.1 \\
$4(\mathrm{~b})$ & $350 \mathrm{MeV}$ & -0.0018 & 1300 & 157.1 \\
$4(\mathrm{c})$ & $300 \mathrm{MeV}$ & -0.0009 & 1300 & 201.1 \\
$4(\mathrm{~d})$ & $100 \mathrm{MeV}$ & 0.0011 & 1300 & 210.5 \\
\hline $5(\mathrm{a})$ & $220 \mathrm{MeV}$ & -0.00048 & 0 & 377.0 \\
$5(\mathrm{~b})$ & $1600 \mathrm{MeV}$ & -0.00112 & 0 & 150.8 \\
$5(\mathrm{c})$ & $0.5 \mathrm{MeV}$ & 0.00201 & 0 & 314.2 \\
$5(\mathrm{~d})$ & $0.095 \mathrm{MeV}$ & 0.0087 & 2300 & 169.6 \\
\hline $6(\mathrm{a})$ & $900 \mathrm{MeV}$ & -0.00061 & 0 & 329.9 \\
$6(\mathrm{~b})$ & $8 \mathrm{MeV}$ & 0 & 0 & 201.1 \\
\hline & & & &
\end{tabular}

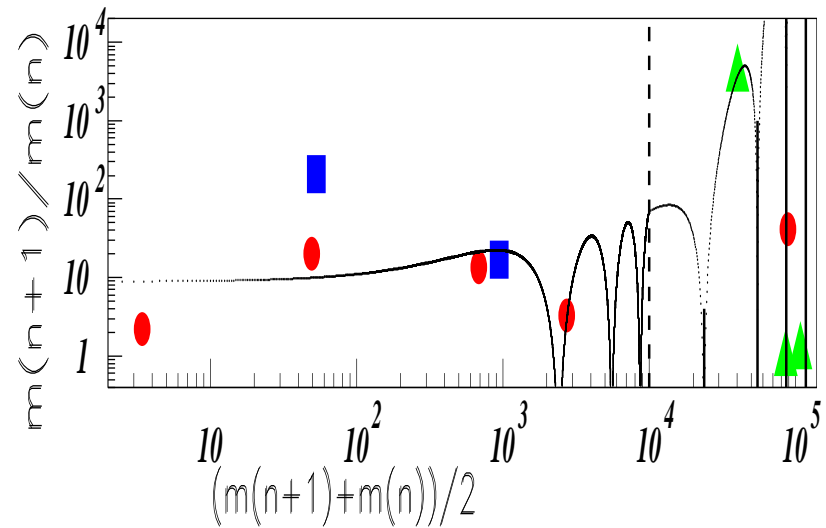

Figure 16. Color on line. The data show the ratios of successive masses of the three fundamental particles versus their corresponding mean masses in $\mathrm{MeV}$. (See text).

\section{References}

1. B. Tatischeff, "Oscillation symmetry applied to: 1) hadronic and nuclei masses and widths 2) astrophysics. And used to predict unknown data.", Proceedings of the $15^{\text {th }}$ International Conference on Nuclear Reaction Mechanisms, Varenna (Italy), p. 35 (2018).

2. B. Tatischeff, "May the oscillation symmetry be applied to TRAPPIST-1 terrestrial planets to predict the mass of the seventh planet ?", Phys Astron Int J. 2(3), 193 (2018). DOI: 10.15406/paij.2018.02.00085.

3. B. Tatischeff, "Oscillation symmetry applied to several astrophysical data. Attempt to predict some properties of the putative ninth and tenth new solar planets", Phys Astron Int J. 2019;3(6):267-274. DOI:10.15406/paij.2019.03.00193. 
Table 2. Parameters describing the fits of figs. 7-16. See text.

\begin{tabular}{|c|c|c|c|c|}
\hline Fig. & $\alpha$ & $\beta\left(\mathrm{MeV}^{-1}\right)$ & $\mathrm{M}_{C}(\mathrm{MeV})$ & $\mathrm{P}(\mathrm{MeV})$ \\
\hline $7(\mathrm{a})$ & $5.5 \mathrm{MeV}$ & -0.2 & 0 & 4.96 \\
\hline $7(\mathrm{~b})$ & $410^{-8} \mathrm{keV}$ & 2.1 & 0 & 3.17 \\
\hline $7(\mathrm{c})$ & $11 \mathrm{MeV}$ & -0.355 & 0 & 1.79 \\
\hline $7(d)$ & $110^{-8} \mathrm{keV}$ & 4.06 & 4.5 & 1.29 \\
\hline $8(\mathrm{a})$ & $0.01 \mathrm{keV}$ & 1.135 & 0 & 1.82 \\
\hline $8(\mathrm{~b})$ & $0.05 \mathrm{keV}$ & 0.73 & 0 & 1.04 \\
\hline $8(\mathrm{c})$ & $0.0044 \mathrm{keV}$ & 0.85 & 0 & 0.69 \\
\hline $9(\mathrm{a})$ & $0.8 \mathrm{MeV}$ & 0 & 0 & 1.31 \\
\hline $9(\mathrm{~b})$ & $3.6 \mathrm{MeV}$ & -0.38 & 0 & 1.51 \\
\hline $9(\mathrm{c})$ & $137 \mathrm{MeV}$ & -0.61 & 0 & 1.76 \\
\hline $9(\mathrm{~d})$ & $1.6 \mathrm{MeV}$ & -0.196 & 0 & 1.43 \\
\hline $10(\mathrm{a})$ & $2.310^{-5} \mathrm{meV}$ & 3.31 & 0 & 0.69 \\
\hline $10(\mathrm{~b})$ & $2.04 \mathrm{meV}$ & -0.085 & 0 & 0.60 \\
\hline $10(\mathrm{c})$ & $110^{-5} \mathrm{meV}$ & 3.18 & 0 & 0.66 \\
\hline $10(d)$ & $0.3 \mathrm{meV}$ & 0.5 & 0 & 0.503 \\
\hline $11(\mathrm{a})$ & $220 \mathrm{MeV}$ & 0 & 0 & 458.7 \\
\hline $11(\mathrm{~b})$ & $4.1 \mathrm{MeV}$ & $-0.032 \mathrm{~A}^{-1}$ & 0 & $4.71 \mathrm{~A}$ \\
\hline $12(\mathrm{a})$ & $0.86 \mathrm{MeV}$ & 0.06 & 0 & 2.01 \\
\hline $12(\mathrm{~b})$ & $2.0 \mathrm{MeV}$ & 0 & 0 & 4.02 \\
\hline $12(\mathrm{c})$ & $2.8 \mathrm{MeV}$ & 0 & 0 & 4.02 \\
\hline $12(d)$ & $4.3 \mathrm{MeV}$ & 0 & 0 & 3.77 \\
\hline $12(\mathrm{e})$ & $5.0 \mathrm{MeV}$ & 0 & 0 & 3.71 \\
\hline $12(\mathrm{f})$ & $2.0 \mathrm{MeV}$ & 0 & 0 & 4.08 \\
\hline $12(\mathrm{~g})$ & $2.7 \mathrm{MeV}$ & 0 & 0 & 2.83 \\
\hline $12(\mathrm{~h})$ & $0.8 \mathrm{MeV}$ & 0.15 & 0 & 3.96 \\
\hline 13 & $2.1 \mathrm{MeV}$ & 0 & 0 & 18.4 \\
\hline $14(\mathrm{a})$ & $95 \mathrm{MeV}$ & 0.0038 & 0 & 301.6 \\
\hline $14(\mathrm{~b})$ & $95 \mathrm{MeV}$ & 0.0038 & 0 & 301.6 \\
\hline $14(\mathrm{c})$ & $95 \mathrm{MeV}$ & 0.0038 & 0 & 301.6 \\
\hline $14(\mathrm{~d})$ & $95 \mathrm{MeV}$ & 0 & 0 & 31064 \\
\hline 15 & 0.008 & 0.000105 & 0 & 18850 \\
\hline 15 & 0.05 & 0.000105 & 0 & 113100 \\
\hline 16 & 10 & 0.00013 & 0 & 3141.59 \\
\hline
\end{tabular}


4. M. Tanabashi et al. (Particle Data Group), Phys. Rev. D 98, 030001 (2018) and 2019 update.

5. Ya-rong Wang et al., "The study of new observed X(2040), X(2240) states and $\omega$ meson family", arXiv:1910.12408v1 [hep-ph] (2019).

6. M. Batra and A. Upadhayay, "Strong decay widths and coupling constants of recent charm meson states", Eur. Phys. J. C 75, 319 (2015).

7. Y. Qin-Tao Song, Dian-Yong Chen, Xiang Liu, and Takayuki, "Charmed-strange mesons revisited: mass spectra and strong decays", Phys. Rev. D 91, 5 (2015).

8. F. Ajzenberg-Selove, 'Energy levels of Light Nuclei A = 13-15', Nucl. Phys. A268, 1 (1976).

9. P.M. Endt, "Energy Levels of A = 21-44 Nuclei (VII)", Nucl. Phys. A521, 1 (1990). DOI:10.1016/03759474(78)90611-5

10. M.J. Martin, Nuclear Data Sheets, 108, 1583 (2007). https://doi:10.1016/j.nds.2007.07.001.

11. O. Hashimoto and H. Tamura, "Spectroscopy of Lambda hypernuclei", Prog. Part. Nucl. Phys. 57, 564 (2006). DOI:10.1016/j.ppnp.2005.07.001.

12. H. Hotchi et al., "Spectroscopy of medium-heavy $\Lambda$ hypernuclei via the $\left(\pi^{+}, K^{+}\right)$reaction", Phys. Rev. C 64, $044302(2001)$.

13. T. Hasegawa et al., "Spectroscopic study of ${ }_{\Lambda}^{10} \mathrm{~B},{ }_{\Lambda}^{12} \mathrm{C},{ }_{\Lambda}^{28} \mathrm{Si},{ }_{\Lambda}^{89} \mathrm{Y},{ }_{\Lambda}^{139} \mathrm{La}$, and ${ }_{\Lambda}^{208} \mathrm{~Pb}$, by $\left(\pi^{+}, K^{+}\right)$reaction", Phys. Rev. 53, 1210 (1996).

14. A.J. Krasznahorkay et al., "Observation of Anomalous Internal Pair Creation in ${ }^{8}$ Be: A Possible Indication of a Light Neutral Boson", Phys. ReV. Lett. 116, 042501 (2016) DOI: 10.1103.

15. T. Walcher, "A simple model to explain narrow nucleon resonances below the $\pi \mathrm{N}$ threshold", arXiv:hep$\mathrm{ph} / 0111279 \mathrm{v} 1$ (2001).

16. A.J. Krasznahorkay et al., "New evidence supporting the existence of the hypothetic X17 particle", arXiv:1910.10459v1 [nucl-ex] Oct 2019. 